\title{
SOME CHAIN CONDITIONS ON WEAK INCIDENCE ALGEBRAS
}

\author{
SURJEET SINGH AND FAWZI AL-THUKAIR
}

Received 19 March 2005 and in revised form 12 July 2005

Let $X$ be any partially ordered set, $R$ any commutative ring, and $T=I^{*}(X, R)$ the weak incidence algebra of $X$ over $R$. Let $Z$ be a finite nonempty subset of $X, L(Z)=\{x \in X$ : $x \leqslant z$ for some $z \in Z\}$, and $M=T e_{Z}$. Various chain conditions on $M$ are investigated. The results so proved are used to construct some classes of right perfect rings that are not left perfect.

\section{Introduction}

Let $R$ be a commutative ring and $X$ a partially ordered set. Let $T=I^{*}(X, R)$ be the set of all functions $f: X \times X \rightarrow R$ such that $f(x, y)=0$, whenever $x \notin y$, and $\{(x, y)$ : $f(x, y) \neq 0$ and $x<y\}$ is finite. Then $T$ is an $R$-algebra under the operations defined as follows. For any $f, g \in T, x, y \in X$, and $r \in R,(f+g)(x, y)=f(x, y)+g(x, y), f g(x, y)=$ $\sum_{x \leq z \leq y} f(x, z) g(z, y)$, and $r f(x, y)=r \cdot f(x, y)$. The algebra $T$ is called weak incidence algebra of $X$ over $R$. For a locally finite partially ordered set $Y$, the concept of incidence algebra $I(Y, R)$ is well known [6]. It can be proved on similar lines as for incidence algebras that for any two partially ordered sets $X, Z$ and any two indecomposable commutative rings $R, S, I^{*}(X, R)$ and $I^{*}(Z, S)$ are isomorphic as rings if and only if $X$ and $Z$ are isomorphic and $R$ and $S$ are isomorphic [5]. It has been seen in [1,5] that weak incidence algebras can be used to construct rings whose left and right maximal rings of quotients need not be isomorphic. Here we give some more such applications. If $X$ is infinite, obviously $T$ is neither left nor right artinian or Noetherian. In the present paper we study chain conditions on a specific one-sided ideal of $T$. Let $Z$ be a finite nonempty subset of $X, L(Z)=\{x \in X: x \leq z$ for some $z \in Z\}$, and $M=T e_{Z}$, where for any subset $Y$ of $X$, $e_{Y} \in T$ is such that $e_{Y}(x, x)=1$ for every $x \in Y$, and $e_{Y}(x, y)=0$ otherwise. Theorem 3.5 shows that $M$ is an artinian left $T$-module if and only if $R$ is artinian and $L(Z)$ satisfies $d c c$ and has no infinite antichain. Theorem 5.2 gives a similar result for $M$ to be Noetherian. In Section 4, the construction of partially ordered sets satisfying $d c c$ but having no infinite antichains is studied. In Section 6, perfect rings are studied, as an application; Theorem 3.5 is used to construct a class of right perfect rings that are not left perfect. 


\section{Preliminaries}

Throughout, all rings have identity element $1 \neq 0$. Let $X$ be a partially ordered set and $R$ a commutative ring. A subset $S$ of $X$ is called an antichain in $X$ if no two members of $S$ are comparable [6]. We will apply the terminology for incidence algebras given in [6] for weak incidence algebras. As usual, for any $x<y$ in $X, e_{x y}$ denotes the corresponding matrix unit in $T=I^{*}(X, R)$. Now $K^{*}(X, R)=\left\{f \in I^{*}(X, R): f(x, x)=0\right.$ for each $\left.x \in X\right\}$ is an ideal $K^{*}(X, R)$ contained in its lower nil radical, and $T / K^{*}(X, R) \cong \Pi_{x \in X} R_{x}$, with each $R_{x}=R$. The following is immediate.

Lemma 2.1. Let $M$ be an artinian (Noetherian) left module over $T=I^{*}(X, R)$ such that $K^{*}(X, R) M=0$, then for some finite subset $Z$ of $X,\left(1-e_{Z}\right) M=0$. In particular, if $M$ is artinian, then $M$ has finite composition length as an $R$-module.

\section{Artinian modules}

A partially ordered set $X$ is said to satisfy strong $d c c$ if it does not contain an infinite sequence $x_{1}, x_{2}, \ldots, x_{n}, \ldots$ such that $x_{j} \ngtr x_{i}$ whenever $i<j$. Let $Z$ be a finite nonempty subset of $X$ and $M=T e_{Z}=\sum_{x \in Z} T e_{x x}$. Now ${ }_{T} M$ is artinian if and only if $T e_{x x}$ is artinian for every $x \in Z$. A finite union of subsets of $X$ satisfies strong $d c c$ if and only if each of the subsets satisfies strong $d c c$. Suppose $M$ is artinian. Then $R$ is artinian. Suppose $L(Z)$ does not satisfy strong $d c c$. Then there exists an $x_{0} \in Z$ such that $L\left(x_{0}\right)$ does not satisfy strong dcc. Therefore there exists an infinite sequence in $L\left(x_{0}\right): x_{1}, x_{2}, \ldots, x_{n}, \ldots$ such that $x_{j} \ngtr x_{i}$ whenever $i<j$. For any $n \geq 1$, let $N_{n}=\sum_{k \geq n} T e_{x_{k} x_{0}}$. Then $N_{n+1} \subset N_{n} \subseteq M$, which contradicts the assumption that $M$ is artinian. Hence $L(Z)$ satisfies strong $d c c$. We now discuss the converse of this result. Henceforth we assume that $R$ is artinian and $L(Z)$ satisfies strong $d c c$. Suppose $M$ is not artinian. Without loss of generality we take $Z=\left\{x_{0}\right\}$ and $M=T e_{x_{0} x_{0}}$. There exists an infinite properly descending chain of $T$-submodules of $M$ : $N_{1} \supset N_{2} \supset \cdots \supset N_{n} \supset \cdots$. For each $i \geq 1$ and $x \in L\left(x_{0}\right)$, let $A_{x}^{(i)}=\left\{a \in R: a e_{x x_{0}} \in N_{i}\right\}$.

Lemma 3.1. (i) $A_{x}^{(i)} \subseteq A_{y}^{(i)}$ whenever $y \leq x$ in $L\left(x_{0}\right)$.

(ii) For any $x \in L\left(x_{0}\right), A_{x}^{(i)} \subseteq A_{x}^{(j)}$ whenever $j \leq i$.

(iii) If $A_{x}^{(i)} \subset A_{y}^{(j)}$, then either $j \leq i$ or $x \nless y$.

(iv) If $A_{x}^{(i)} \nsubseteq A_{y}^{(j)}$, then either $y \nless x$ or $i<j$.

Proof. (i) and (ii) are obvious.

(iii) Suppose $j \nless i$. Then $i<j$, and $A_{x}^{(j)} \subseteq A_{x}^{(i)} \subset A_{y}^{(j)}$. If $x \leq y$, then $A_{y}^{(j)} \subseteq A_{x}^{(j)} \subseteq A_{x}^{(i)}$, which is a contradiction.

(iv) Suppose $y \leq x$. Then $A_{x}^{(j)} \subseteq A_{y}^{(j)}$. If $j \leq i$, then $A_{x}^{(i)} \subseteq A_{x}^{(j)}$, therefore $A_{x}^{(i)} \subseteq A_{y}^{(j)}$, which is a contradiction.

Let $S$ be the set of all $A_{x}^{(i)}$ with $x \in L\left(x_{0}\right)$ and $i \geq 1$. Let $A \in S$. For some $x \in L\left(x_{0}\right)$ and an $i, A=A_{x}^{(i)}$. As $L\left(x_{0}\right)$ satisfies $d c c$, by keeping $i$ fixed we can find $x$ minimal with respect to the pair $(A, i)$. If for some $j>i, A=A_{x}^{(j)}$, then we can find minimal $x^{\prime} \leq x$ for which $A=A_{x^{\prime}}^{(j)}$. Hence we can find an $x \in L\left(x_{0}\right)$ and a positive integer $t$ such that $A=A_{x}^{(t)}$ such that if for some $u \geq t$ and $y \leq x, A=A_{y}^{(u)}$, then $x=y$. Keeping this in mind, a triple 
$(A, t, x)$ is called a critical triple if $A \in S, A=A_{x}^{(t)}$, and if for some $u \geq t, y \leq x, A=A_{y}^{(u)}$, then $y=x$. For any subset $V$ of $S$, the set of those $x \in L\left(x_{0}\right)$ such that $(A, t, x)$ is a critical triple for some $A \in V$ and $t \geqslant 1$ is called the $L\left(x_{0}\right)$-co-support of $V$.

Lemma 3.2. (a) Let $A, B \in S$. If for some positive integer $i,(A, i, x)$ and $(B, i, y)$ are critical triples and $x \neq y$, then one of the following holds: (i) $x<y$ and $B \subset A$, (ii) $y<x$ and $A \subset B$, and (iii) $x$ and $y$ are noncomparable.

(b) If $(A, i, x)$ and $(B, j, y)$ are two critical triples with $A$ and $B$ noncomparable or equal, and $x<y$, then $j<i$.

Proof. (a) is immediate. (b) Now $B=A_{y}^{(j)} \subseteq A_{x}^{(j)}$. If $i \leq j$, then $A_{x}^{(j)} \subseteq A_{x}^{(i)}=A$, therefore $A=B=A_{x}^{(j)}$ and $(A, j, y)$ is a critical pair. But also $A=A_{x}^{(j)}$, hence $x=y$, which is a contradiction. Hence $j<i$.

Lemma 3.3. Let $Y \subseteq S$ be an antichain. Then $Y$ is finite.

Proof. Let $Z$ be the co-support of $Y$. For any $i$, let $Y(i)=\{A \in Y:(A, i, x)$ is a critical triple for some $x \in Z\}$. Let $Z_{i}$ be the set of those $x \in L\left(x_{0}\right)$ such that $(A, i, x)$ is a critical triple for some $A \in Y(i)$. It follows from Lemma 3.2(a) that $Z_{i}$ is an antichain, so $Z_{i}$ is finite. If for some $A, B \in Y(i),(A, i, x)$ and $(B, i, y)$ are critical triples and $A \neq B$, clearly $x \neq y$. Hence $Y(i)$ is finite. Let $Z_{1}$ be the set of minimal members of $Z$. Fix an $x \in Z_{1}$ and a critical triple $(A, k, x)$. Consider any critical triple $(B, i, y)$ with $x<y$ and $B \in Y$. By Lemma 3.2(b), $i<k$. Let $Y_{x}=\{B \in Y$ : there exists a critical triple $(B, i, y)$ with $x \leq y\}$. It follows that $Y_{x}=\bigcup_{i=1}^{k}\left(Y_{x} \cap Y(i)\right)$ is finite. As $Z_{1}$ is finite and $Y=\bigcup_{x \in Z} Y_{x}$, we get $Y$ is finite.

LEMMA 3.4. S is finite.

Proof. For any $A \in S$, the set $S_{A}$ of all those $B \in S$ which are minimal with respect to $A<B$ is finite by Lemma 3.3. Also the set $Y_{1}$ of minimal members of $S$ is finite. After this by using the fact that $R$ has finite composition length, we get $S$ is finite.

THeOREM 3.5. Let $T=I^{*}(X, R)$, where $X$ is any partially ordered set and $R$ is a commutative ring. Let $Z$ be a finite nonempty subset of $X$ and $M=T e_{Z}$. Then $M$ is an artinian left $T$ module if and only if $R$ is artinian and $L(Z)$ satisfies strong $d c c$.

Proof. As remarked earlier it is enough to take $M=T e_{x_{0} x_{0}}$. Suppose that $L\left(x_{0}\right)$ satisfies strong $d c c$ and $R$ is artinian. Suppose $M$ is not artinian. So $M$ has an infinite properly descending chain of $T$-submodules: $N_{1} \supset N_{2} \supset \cdots \supset N_{n} \supset \cdots$. We use the notations given above this result. Let $A \in S$. Fix an $x \in L\left(x_{0}\right)$. Suppose $A=A_{x}^{(i)}$ for some $i$. Then either there exists a smallest positive integer $s_{(x, A)}$ such that $A=A_{x}^{(j)}$ for every $j \geq s_{(x, A)}$ or there exists a largest positive integer $k_{(x, A)}$ such that $A=A_{x}^{\left(k_{(x, A)}\right)}$. Let $Z_{A}$ be the set of those $x \in X$ for which $A$ admits the positive integer $k_{(x, A)}$. Suppose there is no upper bound on $k_{(x, A)}$ as $x$ ranges over $Z_{A}$. So there exists an infinite sequence: $x_{1}, x_{2}, \ldots, x_{n}, \ldots$ in $Z_{A}$ such that $k_{\left(x_{i}, A\right)}>k_{\left(x_{j}, A\right)}$ whenever $i>j$. Then $x_{i} \nless x_{j}$ whenever $i<j$. This contradicts the assumption that $L\left(x_{0}\right)$ satisfies strong $d c c$. Hence there exists a positive integer $k_{A}$ such that $k_{(x, A)}<k_{A}$ for every $x \in Z_{A}$. As $S$ is finite, we can find a positive integer $u$ such that for 
any $A \in S, x \in L\left(x_{0}\right), s(x, A)<u$ and $k_{(x, A)}<u$, whenever $s_{(x, A)}$ or $k_{(x, A)}$ is defined. Consider $N_{u}$. If for some $A_{x}^{(u)}, A_{x}^{(u)} \supset A_{x}^{(u+1)}$, then for $A=A_{x}^{(u+1)}$ we have $k_{(x, A)}>k$ or $s_{(x, A)}>u$, which is a contradiction. Hence $A_{x}^{(u)}=A_{x}^{(u+1)}$. This proves that $N_{k}=N_{k+1}$, which is also a contradiction. Hence $M$ is artinian.

Remark 3.6. Let $X$ be a partially ordered set satisfying strong $d c c$, and $R$ an artinian commutative ring. It follows from the above theorem that for $T=I^{*}(X, R)$, any finitely generated left ideal contained in $A=\sum_{x \in X} T e_{x x}$ satisfies $d c c$. As the ideal $K^{*}(X, R)=\{f \in$ $T: f(x, x)=0$ for every $x \in X\} \subseteq A$, and it is nil, $K^{*}(X, R)$ is right $T$-nilpotent. However this ideal need not be left $T$-nilpotent. For example, let $\mathbb{N}$ be the set of natural numbers with usual ordering. Then for any field $F, K^{*}(\mathbb{N}, F)$ is not left $T$-nilpotent.

\section{Partially ordered sets}

We now prove some results that can help in constructing partially ordered sets satisfying strong $d c c$.

Proposition 4.1. A partially ordered set X satisfies strong dec if and only if it satisfies dcc and it has no infinite antichain.

Proof. If $X$ satisfies strong $d c c$, obviously it cannot have an infinite antichain. Conversely, let $X$ satisfy strong $d c c$ and have no infinite antichain. Suppose there exists an infinite sequence $\left\{x_{i}\right\}$ in $X$ such that $x_{j} \ngtr x_{i}$ whenever $i<j$. These $x_{i}$ are distinct. Let $A$ be the set of these $x_{i}$ and $S$ the set of minimal members of $A$. Then $S$ is a finite nonempty set. So there exists an $x_{i} \in S$ such that $x_{i}<x_{j}$ for infinitely many values of $j$. As a consequence, we can find a $k>i$ such that $x_{i}<x_{k}$, which is a contradiction. Hence $X$ satisfies strong dcc.

Theorem 4.2. Let $X$ and $Y$ be two partially ordered sets satisfying strong dcc, then the partially ordered set $Z=X \times Y$ with the ordering given by $(a, b) \leq(c, d)$ if and only if $a \leq c$ and $b \leq d$ satisfies strong $d c c$.

Proof. That $Z$ satisfies $d c c$ is obvious. Suppose $Z$ has an infinite antichain $S$. Let $A_{1}$ and $A_{2}$ be sets of $X$-components and $Y$-components respectively of the members of $S$. As $Y$ does not contain an infinite antichain, for any fixed $x \in A_{1}$, there are only finitely many $y \in A_{2}$ such that $(x, y) \in S$. Also, the number of minimal members of $A_{1}$ is finite. So there exists a minimal member $x_{1} \in A_{1}$ such that $T_{1}=\left\{(x, y) \in S: x_{1}<x\right\}$ is infinite. Fix an $\left(x_{1}, y_{1}\right) \in S$. If $(x, y) \in T_{1}$, then either $y<y_{1}$ or $y$ and $y_{1}$ are noncomparable. Thus $T_{1}$ satisfies one of the following conditions.

(i) There are infinitely many $(x, y) \in T_{1}$ such that $y<y_{1}$.

(ii) There are infinitely many $(x, y) \in T_{1}$ such that $y$ and $y_{1}$ are noncomparable.

Suppose (i) is satisfied. Then $S_{1}=\left\{(x, y) \in T_{1}: y<y_{1}\right\}$ is infinite. As for $S$, we can find an $\left(x_{2}, y_{2}\right) \in S_{1}$ such that $T_{2}=\left\{(x, y) \in S_{1}: x_{2}<x\right\}$ is infinite. Now $y_{2}<y_{1}$. Suppose $T_{2}$ also satisfies (i), that gives rise to a subset $S_{2}$ analogous to $S_{1}$. Continue the process, and this gives a descending chain in $Y$. As $Y$ satisfies $d c c$, this process must end after a finite number of steps. Thus we get a subset $V_{1}$ of $S_{1}$ and an element $\left(u_{1}, v_{1}\right) \in V_{1}$ such that $V_{2}=\left\{(x, y) \in V: u_{1}<x, y\right.$ and $v_{1}$ are not comparable $\}$ is infinite. Thus for 
any infinite antichain $S$ in $Z$, there exists a $(u, v) \in S$, such that $T=\{(x, y) \in S: u<$ $x, y$ is not comparable with $v\}$ is infinite, so $T$ satisfies (ii). Suppose for some $n \geq 2$, we have constructed infinite sets $V_{i}$ in $S$, for $1 \leq i \leq n,\left(u_{i}, v_{i}\right) \in V_{i}$, for $1 \leq i \leq n-1$ with $V_{i+1}=\left\{(x, y) \in V_{i}: u_{i}<x, v_{i}\right.$ and $y$ are noncomparable $\}$. Now $V_{n}$ has an element $\left(u_{n}, v_{n}\right)$ such that $V_{n+1}=\left\{(x, y) \in V_{n}: u_{n}<x, v_{n}\right.$ and $y$ are noncomparable $\}$ is infinite. This inductive process gives an infinite set $L=\left\{\left(u_{i}, v_{i}\right): i \geq 1\right\} \subseteq S$ such that $u_{i}<u_{i+1}$ for any $i \geq 1$, but $B=\left\{v_{i}: i \geq 1\right\}$ is an infinite antichain in $Y$. This is a contradiction. Hence $Z$ satisfies strong $d c c$.

Example 4.3. For any finite collection of well-ordered sets, their direct product as defined in the above theorem satisfies strong $d c c$.

Definition 4.4. Let $X$ be a partially ordered set satisfying $d c c$. For any nonnegative integer, define $s_{i}(X)$ as follows. Firstly, $s_{0}(X)$ is the set of all minimal elements in $X$. For any $i \geq 0$, an $x \in s_{i+1}(X)$, if it is minimal with respect to the property that for some $y \in s_{i}(X), y<x$. Define $B_{1}(X)=\bigcup_{i \geq 0} s_{i}(X)$.

LEMmA 4.5. Let $X$ be any partially ordered set satisfying $d c c$.

(i) Every $s_{i}(X)$ is an antichain. In addition if $X$ satisfies strong $d c c$, then every $s_{i}(X)$ is finite.

(ii) If for some $i>0$, an $x \in s_{i}(X)$, then there exists a sequence $x_{0}<x_{1}<\cdots<x_{i}=x$ such that $x_{j} \in s_{j}(X)$ for $0 \leq j \leq i$.

(iii) Let $x \in s_{i}(X)$ for some $i, y \in s_{j}(X)$ for some $j>i$. Then $y \nless x$.

Proof. (i) is immediate from the definition and Proposition 4.1.

(ii) follows by using induction on $i$.

(iii) Suppose $y \leq x$. By using (ii) we have $y_{i-1}<y \leq x$. By Definition $4.4, y=x$. At the same time, as $j>i$, by (ii), there exists $z \in s_{i}(X)$ such that $z<y$. This contradicts (i). Hence the result follows.

Definition 4.6. Let $X$ be a partially ordered set satisfying $d c c$. For any ordinal $\alpha$, define $B_{\alpha}(X)$ as follows. $B_{0}(X)=\varnothing$, the empty set, if $\alpha=\beta+1$, then $B_{\alpha}(X)=B_{\beta}(X) \cup$ $B_{1}\left(X \backslash B_{\beta}(X)\right)$. If $\alpha$ is a limit ordinal, then $B_{\alpha}(X)=\bigcup_{\beta<\alpha} B_{\beta}(X)$.

Lemma 4.7. Let $X$ be any partially ordered set satisfying strong dcc.

(i) $B_{1}(X)$ is countable.

(ii) For any two ordinals $\beta<\alpha$, if $\alpha=\beta+\gamma$, then $B_{\alpha}(X)=B_{\beta}(X) \cup B_{\gamma}\left(X \backslash B_{\beta}(X)\right)$.

(iii) $X=B_{\alpha}(X)$ for some ordinal $\alpha$.

(iv) Suppose $X=B_{\alpha}(X)$ for some smallest ordinal $\alpha$. If for every $\beta<\alpha, B_{1}\left(X \backslash B_{\beta}(X)\right)$ is linearly ordered, then $X$ is linearly ordered.

Proof. (i) is immediate from Lemma 4.5.

(ii) follows from Definition 4.4 by using transfinite induction on $\gamma$.

(iii) If $X=B_{1}(X)$, there is nothing to prove. Suppose $X \neq B_{1}(X)$. Then $B_{1}(X)$ is countably infinite. It follows from the definition of a $B_{\beta}(X)$ that if $X \neq B_{\beta}(X)$, then $\left|B_{\beta}(X)\right| \geq|\beta|$. Now there exists a smallest ordinal $\beta$ such that $|\beta|>|X|$. Then $X=B_{\beta}(X)$. Finally (iv) is obvious. 
Remark 4.8. Let $X$ be a partially ordered set satisfying strong $d c c$. If $X$ is infinite, then each $s_{i}(X)$ is nonempty and $B_{1}(X)$ is countably infinite. So, the given ordering on $B_{1}(X)$ can be extended to a linear ordering such that $B_{1}(X)$ becomes isomorphic to the set of natural numbers. Now extend the ordering on $X$ as follows. Let $x, y \in X$. If $x \in B_{\alpha}(X)$ and $y \in B_{\beta}(X)$ such that $\alpha<\beta$ and $y \notin B_{\alpha}(X)$, then set $x<y$. For any ordinal $\alpha$, extend the ordering on $B_{1}(X) \backslash B_{\alpha}(X)$, such that it embeds in the set of natural numbers. This makes $X$ a linearly ordered set satisfying $d c c$. The order on any partially ordered set can be extended to a linear order, [3, Chapter 1]. Here, we see that $X$ can be made into a wellordered set. Let $(Y, \leqslant)$ be any linearly ordered set with the following properties. (i) For some ordinal $\alpha, Y$ is a union of an ascending chain of subsets $\left\{Y_{\beta}\right\}_{\beta \leq \alpha}$, with each $Y_{\beta+1} \backslash Y_{\beta}$ embeddable in the set of natural numbers. (ii) For any limit ordinal $\beta \leq \alpha, Y_{\beta}=\bigcup_{\gamma<\beta} Y \gamma$. (iii) For any $y \in Y \backslash Y_{\beta}$ and $x \in Y_{\beta}, x<y$. Then $Y$ satisfies $d c c$. For each $\beta<\alpha$, consider any ordering $\leqslant_{\beta}$ on $Y_{\beta+1} \backslash Y_{\beta}$ under which $Y_{\beta+1} \backslash Y_{\beta}$ satisfies strong $d c c$ and the given ordering on $Y_{\beta+1} \backslash Y_{\beta}$ extends $\leqslant \beta$. This defines an ordering $\leqslant^{\prime}$ on $Y$ that for each $\beta<\alpha$ coincides with $\leqslant \beta$ on $Y_{\beta+1} \backslash Y_{\beta}$ and equals $\leqslant$ otherwise. Then $\left(Y, \leqslant^{\prime}\right)$ satisfies strong $d c c$.

\section{Noetherian modules}

Let $X$ be a partially ordered set. $X$ is said to satisfy strong acc if it does not contain an infinite sequence $x_{1}, x_{2}, \ldots, x_{n}, \ldots$ such that $x_{j} \nless x_{i}$ whenever $j>i$.

As in Section 3, we consider $M=T e_{Z}$, where $Z$ is a finite nonempty subset of $X$. If $T_{T} M$ is Noetherian, it follows on similar lines as in Section 3 that $\mathrm{R}$ is Noetherian and $L(Z)$ satisfies strong acc.

To prove the converse of the above remark, throughout we take $R$ to be Noetherian, $Z=\left\{x_{0}\right\}$, and $x_{0} \in X$ such that $L\left(x_{0}\right)$ satisfies strong acc. Let $N$ be a submodule of $M$. For each $x \in L\left(x_{0}\right)$, set $A_{x}=\left\{a \in R: a e_{x x_{0}} \in N\right\}$. Each $A_{x}$ is an ideal of $R$ and $N=\sum_{x \in L\left(x_{0}\right)} A_{x} e_{x x_{0}}$. For $x \leq y$ in $L\left(x_{0}\right), A_{y} \subseteq A_{x}$. Let $S$ be the set of all $A_{x}, x \in L\left(x_{0}\right)$. Consider any subset $K$ of $S$. For any $A \in K$, as $L\left(x_{0}\right)$ satisfies $a c c$, we can find $x \in L\left(x_{0}\right)$ maximal with respect to the property that $A=A_{x}$. Let $Z(K)$ be the set of all such maximal elements of $L\left(x_{0}\right)$.

Lemma 5.1. Let $Y \subseteq S$ be an antichain. Then $Z(Y)$ is an antichain and $Y$ is finite.

Proof. Let $x, y \in Z(Y)$ such that $x \leq y$. For some $A, B \in Y, A=A_{x}$ and $B=A_{y}$. However $A_{y} \subseteq A_{x}$, so $A=B$. As $x$ is maximal with respect to $A$, we get $x=y$. Hence $Z(Y)$ is an antichain, so $Z(Y)$ is finite. For each $A \in Y$, there exists an $x \in Z(Y)$ such that $A=A_{x}$. Thus there exists a mapping of $Z(Y)$ onto $Y$. Hence $Y$ is finite.

Theorem 5.2. Let $T=I^{*}(X, R)$ where $X$ is a partially ordered set and $Z$ is a finite nonempty subset of $X$. Then $M=T e_{Z}$ is a Noetherian T-module if and only if $R$ is Noetherian and $L\left(x_{0}\right)$ satisfies strong acc.

Proof. Without loss of generality we take $Z=\left\{x_{0}\right\}$. We use notations given above Lemma 5.1. Let $R$ be Noetherian and $L\left(x_{0}\right)$ satisfy strong acc. Let $N$ be a $T$-submodule of $M$. As $R$ is Noetherian, $Y_{1}=\{A \in S: A$ is maximal in $S\}$ is nonempty and no two members of $Y_{1}$ are comparable. Set $Z_{1}=Z\left(Y_{1}\right)$. Consider $N_{1}=\sum_{x \in Z_{1}} T A_{x} e_{x x_{0}}$. Let $y \leq x$ with $x \in Z_{1}$, then $A_{x} \subseteq A_{y}$, therefore $A_{y}=A_{x}$ and $A_{y} e_{y x_{0}}=e_{y x}\left(A_{x} e_{x x_{0}}\right) \subseteq N_{1}$. Hence $N_{1}=\sum_{x \in L\left(Z_{1}\right)} A_{x} e_{x x_{0}}$. Suppose, for some $n \geq 1$, we have already defined subsets $Z_{1}, Z_{2}, \ldots, Z_{n}, V_{n}=\bigcup_{i=1}^{n} Z_{i}$, and 
$N_{n}=\sum_{x \in V_{n}} T A_{x} e_{x x_{0}}$ such that the following hold. (i) $N_{n}=\sum_{x \in L\left(V_{n}\right)} A_{x} e_{x x_{0}}$, (ii) for any $y \in L\left(V_{n}\right)$, there exists an $x$ in $V_{n}$ such that $y \leq x$ and $A_{y}=A_{x_{i}}$, and (iii) for any $y \in$ $L\left(x_{0}\right) \backslash L\left(V_{n}\right)$, there exists $x \in Z_{n}$ such that $A_{y}<A_{x}$. Set $S_{n+1}=\left\{A \in S: A=A_{x}\right.$ for some $\left.x \in L\left(x_{0}\right) \backslash L\left(V_{n}\right)\right\}$ and $Y_{n+1}$ the set of all maximal members of $S_{n+1}$. Set $Z_{n+1}=Z\left(Y_{n+1}\right)$, $V_{n+1}=V_{n} \cup Z_{n+1}$, and $N_{n+1}=\sum_{x \in V_{n}} T A_{x} e_{x x_{0}}$. The above three conditions are obviously satisfied by $N_{1}$. Suppose they are satisfied by $N_{n}$ for some $n$. Suppose $y \in Z_{n+1}$ and $x \in X$ such that $x<y$. Then $A_{y} \subseteq A_{x}$. If $x \notin L\left(V_{n}\right), A_{x}=A_{y}$. If $y \in L\left(V_{n}\right)$, by (ii) there exists $z \in V_{n} \subseteq V_{n+1}$ such that $y \leq z$ and $A_{y}=A_{z}$. Hence $N_{n+1}=\sum_{x \in L\left(V_{n+1}\right)} A_{x} e_{x x_{0}}$. Thus $N_{n+1}$ satisfies (i), (ii), and (iii). For each $i$ for which $Z_{i}$ is non-empty, fix an $x_{i} \in Z_{i}$. If an $L\left(Z_{i}\right) \neq \mathrm{S}$, obviously $Z_{i+1} \neq \varnothing$. For $i<j$, as $L\left(V_{i}\right) \cap Z_{j}=\varnothing, x_{j} \nless x_{i}$. As $L\left(x_{0}\right)$ satisfies strong acc, it follows that there exists an $n$ such that $Z_{n} \neq \varnothing$ but $Z_{n+1}=\varnothing$. Consequently, $L\left(x_{0}\right)=L\left(V_{n}\right), N_{n}=N$. As $V_{n}$ is finite, each $A_{x}$ is finitely generated as an $R$-module, and $N=\sum_{x \in V_{n}} T A_{x} e_{x x_{0}}$, it follows that $N$ is a finitely generated $T$-module. Hence $M$ is Noetherian.

Remark 5.3. Let $X^{\prime}$ be the dual of a partially ordered set $X$. For any commutative ring $R$, set $T^{\prime}=I^{*}\left(X^{\prime}, R\right)$ and $T=I^{*}(X, R)$. These two algebras are naturally anti-isomorphic. Let $Z$ be a finite nonempty subset of $X, M=e_{Z} T$, and $U(Z)=\{x \in X: x \geq z$ for some $z \in$ $Z$ \}. By using the anti-isomorphism between $T$ and $T^{\prime}$ and Theorems 3.5 and 5.2, we get the following results:

(i) $M_{T}$ is artinian if and only if $R$ is artinian and $U(Z)$ satisfies strong acc;

(ii) $M_{T}$ is Noetherian if and only if $R$ is Noetherian and $U(Z)$ satisfies strong $d c c$.

Remark 5.4. Let $X$ be a locally finite partially ordered set, and $T=I(X, R)$ the incidence algebra of $X$ over a commutative ring $R$. Suppose $R$ is artinian and for some $x_{0} \in X$, $L\left(x_{0}\right)$ satisfies strong $d c c$. As $L\left(x_{0}\right)$ has finitely many minimal elements, $L\left(x_{0}\right)$ is a finite set, so $M=T e_{x_{0} x_{0}}$, being a finite direct sum of copies of $R$, is trivially an artininian left $T$-module. Hence $M$ is an artinian left $T$-module if and only if $R$ is artinian and $L\left(x_{0}\right)$ satisfies strong $d c c$.

Now suppose $R$ is Noetherian, $L\left(x_{0}\right)$ satisfies strong $a c c$, and $N$ is a $T$-submodule of $M$. As in the proof of Theorem 5.2, we have $Y_{1}$ and $Z_{1}=Z\left(Y_{1}\right)$. Consider $N_{n}$ as defined in the proof of Theorem 5.2. Now $N_{1}=\sum_{x \in Z_{1}} T A_{x} e_{x x_{0}}$. For any $x \in Z_{1}$, let $G_{x}=\left\{b_{x j}\right.$ : $\left.1 \leqslant j \leqslant n_{x}\right\}$ generate $A_{x}$ as an $R$-module. Consider any $f \in N_{1}$ with $D_{f}=\left\{z \in L\left(x_{0}\right)\right.$ : $\left.f\left(z, x_{0}\right) \neq 0\right\} \subseteq L\left(Z_{1}\right)$. Let $z \in L\left(Z_{1}\right)$. Then for any $x \in Z_{1}, A_{z}=A_{x}$ whenever $z \leqslant x$. So $f\left(z, x_{0}\right)=\sum_{z \leqslant x} \sum_{j=1}^{n_{x}} r_{z x j} b_{x j}$, where $x \in Z_{1}$. Then the formal sum $g_{x j}=\sum_{z \leqslant x} r_{z x j} e_{z x} \in T$ and $f=\sum_{x \in Z_{1}} \sum_{j=1}^{n_{x}} g_{x j} b_{x j} e_{x x_{0}} \in N_{1}$. Hence $N_{1}=\left\{f \in N_{1}: D_{f} \subseteq L\left(Z_{1}\right)\right\}$. Inductively, one can prove that for any $n \geqslant 1, N_{n}=\left\{f \in N: D_{f} \subseteq L\left(V_{n}\right)\right\}$. Each $N_{n}$ is finitely generated. Hence as in Theorem 5.2, we get that $N=N_{n}$ for some n, hence $N$ is finitely generated. This proves that $M$ is a Noetherian left $T$-module if and only if $R$ is Noetherian and $L\left(x_{0}\right)$ satisfies strong acc.

\section{Perfect rings}

A partially ordered set $X$ is said to locally satisfy strong $d c c$, if for any finite subset $S$ of $X, L(S)$ satisfies strong $d c c$. Throughout, $R$ is an artinian, commutative local 
ring, $X$ is a partially ordered set locally satisfying strong $d c c$, and $T=I^{*}(X, R)$. Let $T^{\prime}=R+K^{*}(X, R)$. Then $T^{\prime}$ is a local ring. We will prove that $T^{\prime}$ is right perfect. We will write $K$ for $K^{*}(X, R)$.

Lemma 6.1. Any finitely generated left ideal of $T^{\prime}$ contained in $K^{*}$ is artinian.

Proof. Let ${ }_{T} C$ be any artinian module. By Lemma 2.1, $C / K^{*} C$ is of finite composition length over $R$. Let $A=\sum_{i=1}^{n} T^{\prime} b_{i}$ be a finitely generated left ideal of $T^{\prime}$ contained in $K^{*}$. Then $B=\sum_{i=1}^{n} T b_{i}$ is a finitely generated left ideal of $T$ contained in $K^{*}$. Let $A=$ $A_{1} \supseteq A_{2} \supseteq \cdots \supseteq A_{n} \supseteq \cdots$ be a descending chain of left ideals of $T^{\prime}$. As ${ }_{T} B$ is artinian, there exists a positive integer $m$ such that $K^{*} A_{i}=K^{*} A_{m}$ for any $i \geq m$. Let $B_{i}=T A_{i}$. Then $K^{*} B_{i}=K^{*} A_{i}$. Now $B_{i}$ is an artinian left $T$-module. It follows that for any $i \geq m$, $B_{i} / K^{*} B_{i}$ is of finite composition length over $R$. Therefore there exists an $n \geq m$ such that $A_{j} / K^{*} A_{j}=A_{j} / K^{*} A_{n}=A_{n} / K^{*} A_{n}$ for any $j \geq n$. Hence $A_{j}=A_{n}$ for any $j \geq n$.

Theorem 6.2. $T^{\prime}$ is a local, right perfect ring.

Proof. It is enough to prove that $T^{\prime}$ satisfies $d c c$ on principal left ideals [2, Theorem 28.4]. Let $A_{1} \supseteq A_{2} \supseteq \cdots \supseteq A_{n} \supseteq \cdots$ be a descending chain of principal left ideals of $T^{\prime}$. In view of Lemma 6.1, we take $A_{i}=T^{\prime}\left(\alpha_{i} I+b_{i}\right)$ for some $\alpha_{i} \neq 0$ in $R$ and $b_{i} \in K^{*}$. Then $\alpha_{i+1} I+b_{i+1}=\left(\beta_{i} I+c_{i}\right)\left(\alpha_{i} I+b_{i}\right)$ for some $\beta_{i} \in R$, and $c_{i} \in K^{*}$. This gives $\alpha_{i+1}=\beta_{i} \alpha_{i}$ and $a n n_{R}\left(\alpha_{i}\right) \subseteq a n n_{R}\left(\alpha_{i+1}\right)$. As $R$ is Noetherian, there exists a positive integer $m$ such that $a n n_{R}\left(\alpha_{i}\right)=a n n_{R}\left(\alpha_{i+1}\right)$ for any $i \geq m$. Therefore $\beta_{i}$ is a unit for any $i \geq m$ and $\beta_{i} I+c_{i}$ is a unit. Hence $A_{i}=A_{m}$ for any $i \geq m$.

The dualization of the above result gives the following.

Theorem 6.3. Let $X$ be a partially ordered set such that for any finite nonempty subset $Z$ of $X, U(Z)$ satisfies strong acc, $R$ is an artinian commutative ring, and $T=I^{*}(X, R)$. Then $T^{\prime}=R+K^{*}(X, R)$ is left perfect.

Examples of rings that are right perfect but not left perfect are well known (one such example is the dual of example given in [2, Exercise 2, page 322]). By using the above theorem, we end this section by constructing a class of right perfect rings that are not left perfect.

Example 6.4. Let $X$ be any partially ordered set that locally satisfies strong dcc, but has a finite, nonempty subset $Z$ such that $L(Z)$ is not finite. As $L(Z)$ satisfies strong $d c c, L(Z)$ has a subset $V$ isomorphic to the set of natural number. Any infinite wellordered set not embeddable in the set of natural numbers is such a set $X$. Thus $V$ is given by elements: $x_{1}<x_{2}<\cdots<x_{n}<\cdots$. Let $R$ be a local artinian ring, and $T^{\prime}=$ $R+K^{*}(X, R)$. By Theorem 6.2, $T^{\prime}$ is right perfect, however $\left\{e_{x_{1} x_{i}} T^{\prime}\right\}_{i \geq 2}$ is an infinite, nonterminating descending sequence of principal right ideals in $T^{\prime}$. Hence $T^{\prime}$ is not left perfect.

\section{Acknowledgment}

The authors are thankful to the referees for their comments and suggestions. 


\section{References}

[1] F. Al-Thukair, S. Singh, and I. Zaguia, Maximal ring of quotients of an incidence algebra, Arch. Math. (Basel) 80 (2003), no. 4, 358-362.

[2] F. W. Anderson and K. R. Fuller, Rings and Categories of Modules, Graduate Texts in Mathematics, vol. 13, Springer, New York, 1974.

[3] P. C. Fishburn, Interval Orders and Interval Graphs, Wiley-Interscience Series in Discrete Mathematics, John Wiley \& Sons, New York, 1985.

[4] T. Y. Lam, Lectures on Modules and Rings, Graduate Texts in Mathematics, vol. 189, Springer, New York, 1999.

[5] S. Singh and F. Al-Thukair, Weak incidence algebra and maximal ring of quotients, Int. J. Math. Math. Sci. 2004 (2004), no. 53, 2835-2845.

[6] E. Spiegel and C. J. O'Donnell, Incidence Algebras, Monographs and Textbooks in Pure and Applied Mathematics, vol. 206, Marcel Dekker, New York, 1997.

Surjeet Singh: Department of Mathematics, King Saud University, P.O. Box 2455, Riyadh 11451, Kingdom of Saudi Arabia

E-mail address: ssingh@ksu.edu.sa

Fawzi Al-Thukair: Department of Mathematics, King Saud University, P.O. Box 2455, Riyadh 11451, Kingdom of Saudi Arabia

E-mail address: thukair@ksu.edu.sa 


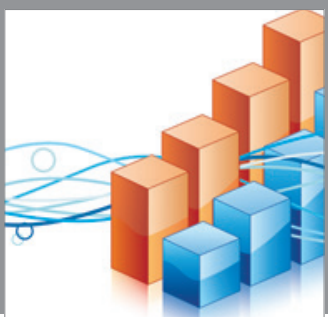

Advances in

Operations Research

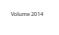

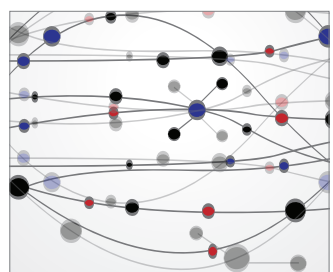

\section{The Scientific} World Journal
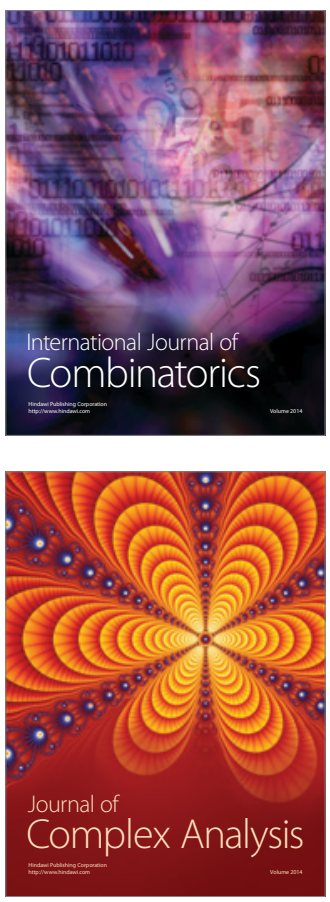

International Journal of

Mathematics and

Mathematical

Sciences
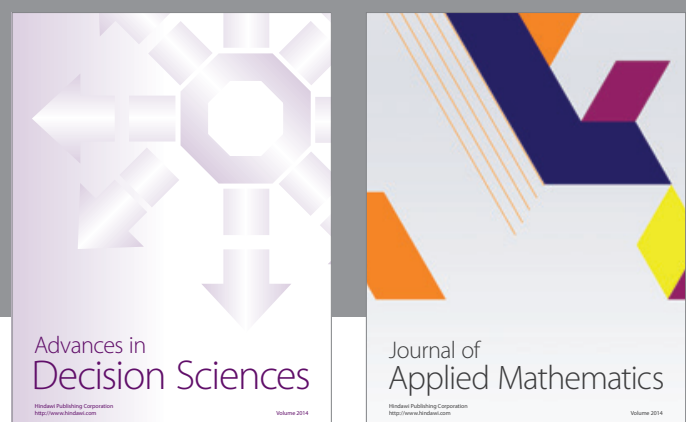

Journal of

Applied Mathematics
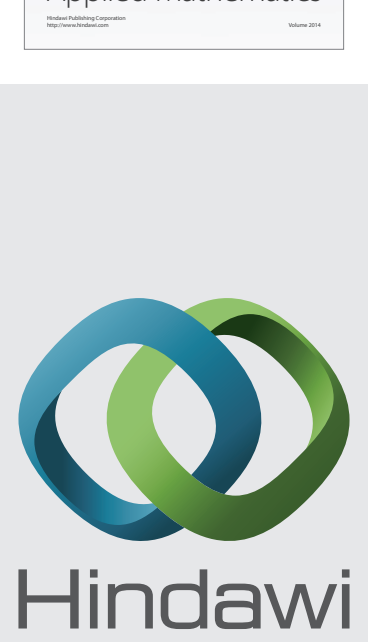

Submit your manuscripts at http://www.hindawi.com
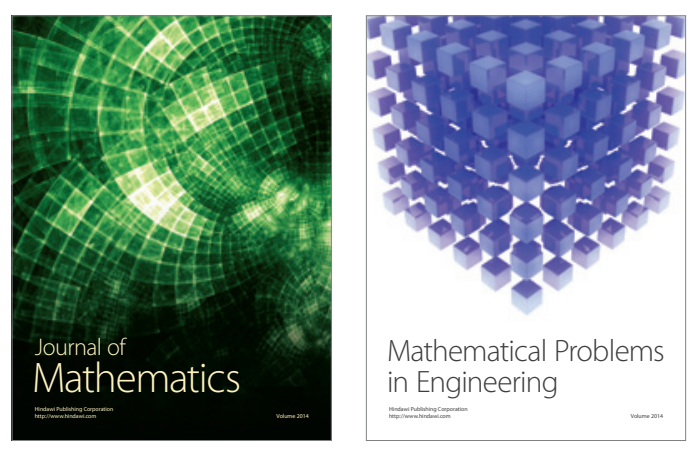

Mathematical Problems in Engineering
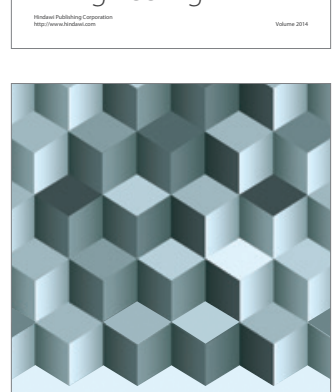

Journal of

Function Spaces
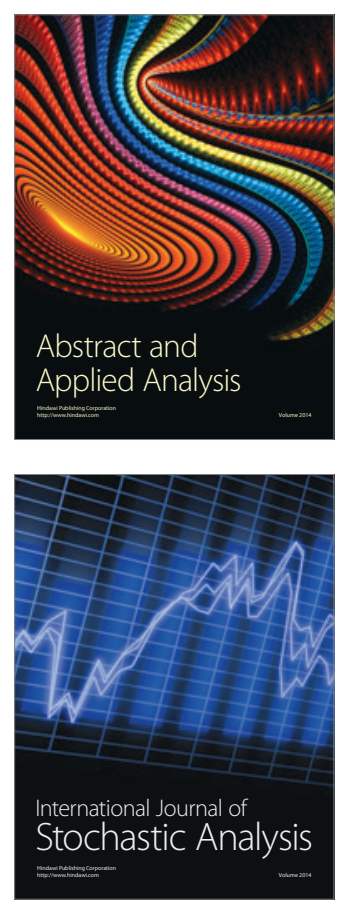

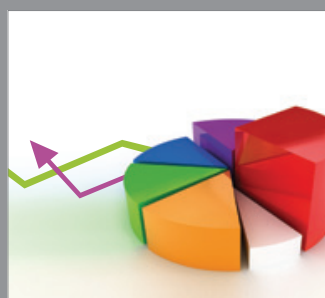

ournal of

Probability and Statistics

Promensencen
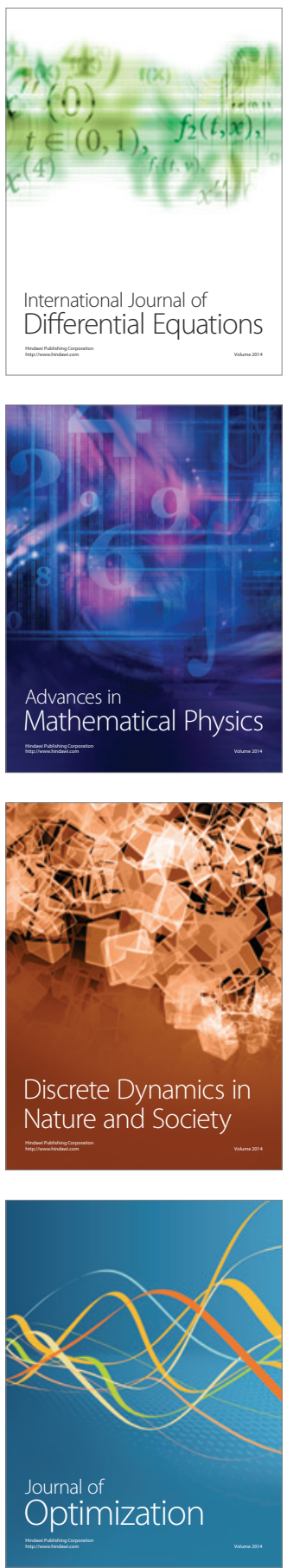